\title{
PENSAR LAS CIUDADES DESDE LA GEOGRAFÍA
}

\author{
Carmen Delgado Viñas \\ Departamento de Geografía, Urbanismo y Ordenación del Territorio \\ Universidad de Cantabria
}

\section{RESUMEN}

En este texto se pasa revista a la evolución de la investigación que la Geografía ha hecho sobre la ciudad, en tanto que espacio físico o urbs, desde principios del siglo xx hasta la actualidad. Esta revisión y puesta al día se centra en el trabajo realizado por investigadores españoles pero se apoya también en la dinámica epistemológica y metodológica de los estudios urbanos y urbanísticos a nivel internacional, fundamentalmente europeo, puesto que el trabajo de los autores españoles ha ido siguiendo y enmarcándose en los paradigmas y métodos predominantes en nuestro entorno académico y científico próximo. A causa de las obligadas limitaciones de extensión, las referencias aportadas son necesariamente incompletas y sólo pretenden aproximarse a una modesta puesta al día del estado de la cuestión, alejada de cualquier pretensión de una exhaustividad que es casi inalcanzable al referirse a un campo de estudio tan dilatado y complejo y abordado por tantas disciplinas científicas simultáneamente.

Palabras clave: Geografía urbana, Historia urbana, Urbanismo, ciudades, espacio urbano.

\section{ABSTRACT}

In this paper is analyzed the research developments that Geography has done about cities, like urbs or physical space, since the early twentieth century to the present. Both the review and the update focus on the studies conducted by the Spanish researchers, but also relies on the epistemological and methodological dynamics of urban studies at the international level, mainly European. Because the work of Spanish authors has been following and framing itself in the predominant paradigms and methods of our next scientific and academic environment.

Due to necessary restrictions in the extension of an article, the references provided are necessarily incomplete and are only intended to be a modest update of the state of the question, far from any pretense of comprehensiveness that is almost unattainable for the 
research referring to a field so vast and complicated and approached by many scientific disciplines simultaneously.

Keywords: Urban Geography, Urban History, Urbanism, cities, urban space.

«la ciudad y la forma de paisaje que ella instrumenta,

en su forma extrema y límite,

significa la operación trasmutadora más radical

llevada a cabo por el hombre en el medio natural»

(Manuel de Terán, 1966:165)

\section{EL ANÁLISIS DE LOS ESPACIOS URBANOS}

He querido iniciar este texto con las palabras de un eminente estudioso de la ciudad, maestro de geógrafos e historiador, que supo ver el espacio urbano con una mirada transdisciplinar en la que se aunaban varios de los enfoques (temporales, espaciales y arquitectónicos), que son inexcusables, en mi opinión, para abordar el estudio de la ciudad en clave de Geografía urbana.

La Geografía, cuyo objeto de estudio es el territorio en su totalidad, ha encontrado en la ciudad, y en las dificultades para aprehenderla, un atrayente desafío (Straszewicz, 1981). Por ello, Manuel de Terán consideraba que, para comprender el fenómeno urbano en su integridad, es imprescindible que exista un diálogo permanente entre diferentes disciplinas ya que la ciudad es la expresión, materializada espacialmente, de complejos procesos socioeconómicos, sin olvidar, además, la importancia que tiene el medio natural como condicionante de la forma urbana. Como ha afirmado recientemente uno de sus discípulos, Francisco Quirós, Terán introdujo la consideración del espacio urbano como un producto espacial, resultante de un proceso temporal cuyo desarrollo es imprescindible considerar para poder comprender las huellas del pasado, impresas en los paisajes urbanos actuales. Igualmente, H. Capel (2010) considera que la perspectiva social de la Geografía ha sido, casi desde su origen y ante todo, una Geografía de la ciudad puesto que, desde paradigmas distintos, se ha venido considerando que las ciudades son productos espaciales de los diferentes tipos de sociedad que las construyen y, en consecuencia, reflejan espacialmente la organización social en toda su complejidad y en todas sus relaciones.

Varias disciplinas científicas han realizado diversas aproximaciones al estudio de la ciudad, pero prácticamente ninguna abarca una perspectiva total del fenómeno urbano, sino que cada una aborda las ciudades bajo un ángulo particular. Una situación que es comprensible si aceptamos que «La ciudad es, manifiestamente, algo muy complicado» (Harvey, 1977:15); que el hecho urbano es un objeto diverso que requiere un análisis múltiple y pluridisciplinar 
para explicar una realidad dinámica y compuesta de innumerables facetas, sobrepuestas y contrapuestas. Es por ello que en alguna ocasión han surgido iniciativas, frustradas hasta ahora, para fundar una disciplina dedicada exclusivamente al estudio específico del espacio urbano, a modo de ciencia urbanística total, como la propuesta denominada «civics» de Patrick Geddes (1915), la del «urbanismo» de M. Lhéritier (1921) o la más reciente «new science of cities» de Michael Batty (2013) (Juaristi, 2015).

En cualquier caso, y a pesar del predominio de los enfoques necesariamente sectoriales y parciales, los profesionales de todas las disciplinas que tienen como objeto de análisis la ciudad, coinciden desde hace mucho tiempo en lugares de interés comunes en los que los intercambios cognitivos y metodológicos son obligados. Así, la arquitectura, la economía, la geografía, la historia, la ingeniería, la sociología y algunas disciplinas o áreas de trabajo científico más, han realizado aproximaciones al estudio de lo urbano a partir de variados enfoques. Similares planteamientos sostienen muchos autores para quienes la única forma de entender la historia urbana es la integración de múltiples ciencias que ayudan a explicar la ciudad, que «es una estructura compleja que no puede reducirse al mínimo común denominador.» (Magnago, 2003:51).

No obstante, algunas disciplinas tienen una vocación integradora más clara de aproximación al conjunto del espacio urbano. Este es el caso de la Geografía Urbana y el Urbanismo, aunque no sean las únicas que utilizan el enfoque integral y a pesar de que existan diferencias conceptuales y metodológicas entre ambas.

En general, se admite que el Urbanismo es una disciplina y práctica social, que posee un carácter eminentemente técnico, cuya finalidad principal es la reforma y ordenación del crecimiento de los espacios urbanos de acuerdo con las necesidades materiales y sociales de sus habitantes. Por su parte, la mayoría de las definiciones de Geografía urbana coinciden en afirmar que es la rama de la Geografía que estudia la ciudad desde el punto de vista espacial, como entidad per se y como elemento integrante de una red o sistema territorial de asentamientos, tanto desde la perspectiva de la dinámica presente como bajo el enfoque de la evolución y los procesos históricos que han configurado las ciudades y las redes urbanas. Un conocimiento empírico de las realidades urbanas, del que derivan patrones y modelos teóricos propositivos, estructurales y morfológicos, que proporciona de manera eficaz la Geografía Urbana y que sirven de fundamento y soporte teóricos de la intervención urbanística.

Por otro lado, la Historia y la Geografía han cruzado sus miradas desde hace largo tiempo sobre el estudio de los espacios urbanos, tópico común para historiadores y geógrafos pero bajo enfoques parcialmente distintos. Como no podía ser de otra forma, ambas ciencias han venido prestándose apoyo mutuo para abordar el análisis de la ciudad. Un método de trabajo coherente con la 
aseveración que a principios del siglo xx, hacía el insigne geógrafo Élisée Reclus: «la Geografía es la Historia en el espacio del mismo modo que la Historia es la Geografía en el tiempo». Tal y como afirman algunos autores, la mayor coincidencia es la que se produce específicamente entre la geografía histórica y la arqueología del espacio de cuya unión nace una «arqueología urbana» dedicada a descubrir la historia espacial de la ciudad a partir de la colaboración interdisciplinar entre geógrafos, arquitectos, urbanistas e historiadores.

Habitualmente los historiadores, poniendo el acento en la dimensión temporal, no han considerado lo urbano, en un sentido espacial, como materia central de sus preocupaciones, sino que han concebido la ciudad, ante todo, como contexto físico o escenario en el que se producen las grandes transformaciones contemporáneas, el lugar en que se desenvuelven los grupos sociales y se desarrollan los hechos históricos. Sin embargo, se puede constatar que en las dos últimas décadas, entre los siglos Xx y XxI, ha surgido un nuevo cuerpo de trabajos históricos en los estudios urbanos que está reconfigurando el campo de la historia urbana de manera gradual (Gunn, 2013). De forma preferente estos estudios históricos urbanos se han centrado en un triple objetivo: el crecimiento de la ciudad y la expansión del espacio urbano, la construcción de las infraestructuras y servicios públicos y los cambios en las instituciones de gobierno local, sobre todo en lo que respecta a las competencias relacionadas con la planificación urbana, en particular durante el proceso de tránsito de la ciudad moderna a la contemporánea lo que, en el caso español, implica abordar un intervalo cronológico de unos tres siglos, desde comienzos del XVIII a finales del Xx. Como es lógico, en ese ámbito temático y temporal se ha producido la confluencia de historiadores, geógrafos y urbanistas, con objetos de investigación, métodos de trabajo y fundamentaciones conceptuales parcialmente diversos.

Por regla general, los geógrafos, insistiendo en la variable territorial, han concebido el espacio urbano como objeto en sí mismo, como elemento autónomo y activo, en continua construcción y reconstrucción al compás de las mutaciones socioeconómicas históricas. Una perspectiva que, por cierto, no se encuentra muy alejada de la forma de comprender la Historia urbana a partir del entendimiento de «la relación dialéctica entre la construcción de la ciudad y las realidades sociales, económicas, políticas y culturales» que intervienen en su configuración (Bahamonde, 1993), aunque enfocando esencialmente, en el caso de la Geografía, al componente espacial y a los agentes y elementos urbanísticos que participan en la construcción material de la ciudad; los mismos que la dotan, como ente físico, de una estructura funcional, una forma, una fisonomía y un paisaje determinados.

El punto de partida de esta concepción es que los espacios urbanos, como otros espacios geográficos, son productos sociales y, por tanto, están sometidos a las condicionantes históricos, por lo que es preciso analizarlos en el contexto 
en que se han configurado, que es el de la formación social histórica correspondiente. El espacio urbano es entendido como un producto que se forma y evoluciona bajo las mismas leyes que rigen el sistema social que lo construye, como una estructura socioespacial, un subsistema, que forma parte de un sistema territorial más amplio.

Partiendo de la premisa de que los principales cambios y transformaciones de la sociedad se reflejan en el espacio urbano, este punto de vista obliga también a analizar el espacio en clave temporal para comprender las estructuras y formas pasadas y presentes de la ciudad. Se trata de una forma de aproximación a lo urbano, recientemente renovada, aunque de larga trayectoria entre los geógrafos, en la que prevalece la óptica espacial sin olvidar, no obstante, el enfoque temporal, por lo que, en consecuencia, cabe calificarla, sin ningún rubor, como historicista.

Álvarez Mora (1996) titulaba un artículo como «La necesaria componente espacial de la Historia Urbana», una aserción que podría complementarse con la imprescindible referencia a «la necesaria componente temporal de la Geografia Urbana». Más aun cuando, desde hace algún tiempo, la labor de los geógrafos urbanos se ha orientado más hacia el análisis de la problemática actual, con mayores posibilidades de aplicación de conocimientos al planeamiento y gestión del espacio urbano, que hacia la tarea de conocer, interpretar y explicar la configuración histórica de las ciudades. Eso ha originado, como es lógico, la disminución del número de los cultivadores de la Geografía urbana histórica. Bien es verdad que, en la actualidad, parece que está rebrotando el interés por el conocimiento del patrimonio arquitectónico y urbanístico, desde el punto de vista de la consideración del espacio urbano como patrimonio cultural y bajo nuevos enfoques metodológicos, en relación con la valoración del paisaje urbano como recurso aprovechable para el desarrollo socioeconómico sostenible de la ciudad.

Muchas voces, procedentes de distintas áreas de conocimiento, están reclamando la necesidad de renovar las miradas o las perspectivas sobre la ciudad contemporánea. Desde la Geografía, H. Capel ha invitado en distintas ocasiones a los historiadores a acercarse a los asuntos actuales de la ciudad (de gestión y gobernanza, cambios morfológicos, estrategias, etc.) para aportar su conocimiento de la problemática del pasado a la solución de los problemas del presente, incluso a la elaboración de propuestas de futuro. Del mismo modo que se considera imprescindible que los geógrafos apliquemos un enfoque histórico para comprender las situaciones actuales.

En definitiva, ninguna disciplina que se allegue al estudio de las ciudades puede obviar las aportaciones que se han hecho desde las demás puesto que el propio concepto de ciudad alude a aspectos diversos (Capel, 2010). Más bien, parece requerir una aproximación «mestiza» donde los bordes y los márgenes 
disciplinares se confunda para ir dando lugar a campos temáticos emergentes (Whithehand, Wolch, Valencia). Probablemente ese enfoque ayudaría a superar los desafíos conceptuales y metodológicos y a prevenir los riesgos del distanciamiento científico; algo que, aunque afortunadamente aún no ha ocurrido, es una amenaza cierta en el contexto de los procesos recientes de remodelación de las disciplinas en las enseñanzas universitarias, que parecen tender a «guardar las distancias» entre las ciencias sociales y territoriales e, incluso, a disolver los vínculos tradicionales entre la Historia y la Geografía.

Tal vez haya llegado el momento de avanzar más deprisa en este sentido y pasar del debate pluridisciplinar y la cooperación multidisciplinar a una simbiosis imprescindible entre la dimensión espacial y la temporal que permita acometer los estudios urbanos a partir de un acercamiento transdisciplinar que aúne perspectivas propias de la Geografía, de la Historia, de la Historia del Arte, de la Historia Económica, del Urbanismo y un largo etcétera. Porque, como ha afirmado recientemente Derek Gregory «cada uno de estos conceptos ('geografía histórica' e 'historia espacial') tiene su propia genealogía, pero es vano privilegiar uno sobre otro. Resulta mucho más productivo, creo, permitir que cada uno se diluya en el contrario» (Elden, Gregory y Sevilla, 2011:94).

En coherencia con las premisas expuestas hasta aquí, en este texto me propongo realizar una breve revisión historiográfica sobre las aproximaciones a la ciudad desde la Geografía Urbana, una de las disciplinas que, en mi opinión, ha perseverado en mayor medida y con un enfoque más integrador en el estudio de la ciudad.

Aunque con una perspectiva necesariamente amplia y plural, el punto de vista principal ha de ser, en mi caso, el geográfico por razones de formación académica, por trayectoria investigadora y por convicción científica apoyada en la distinción que, en los años setenta, hacía H. Capel (1975) al recordar los tres sentidos que puede tener la ciudad (urbs o espacio físico urbano, civitas o sociedad urbana y polis o comunidad política) y señalar que, dentro de ese abanico de opciones, lo específico de la mirada geográfica es el estudio de la ciudad como urbs, pese a que años después (2003) el mismo autor completó acertadamente dicha afirmación declarando que la ciudad debe ser analizada en esos tres niveles simultáneamente.

Y, puesto que la temática es muy amplia y la bibliografía extensísima, trataré de limitar también mis referencias a dar cuenta de las aportaciones de carácter general sobre los espacios urbanos, sobre todo los españoles, prescindiendo de las referencias a los estudios empíricos de carácter local o de caso, salvo que constituyan hitos en la evolución de la historiografía urbana, y que, como corresponde a un campo de trabajo territorialmente concreto, son los más numerosos.

Por otra parte, es imposible hacer una revisión de la evolución de la investigación española sobre las ciudades sin hacer referencia a la dinámica de este 
área de estudio a nivel internacional, fundamentalmente europeo, puesto que el trabajo de los autores españoles ha ido siguiendo y enmarcándose, por lo general con cierto retraso, en los paradigmas y métodos predominantes en su contexto espacial.

Por los motivos expuestos, las referencias aportadas son, necesariamente, incompletas y sólo tratan de ser una modesta puesta al día del «estado del arte» alejada de cualquier pretensión de exhaustividad, que sería casi quimérica al referirse a un objeto o, mejor aún, un campo de estudio tan complejo y abordado por tantas disciplinas científicas. Por otra parte, son muy abundantes las revisiones bibliográficas, epistemológicas y metodológicas, que se han hecho sobre esta mismas temática de forma que ésta no pretende ser más que una continuación actualizada y apoyada en buena parte de aquéllas.

Desde los años ochenta, incluso mucho antes aunque sea excepcional (Aurousseau, 1924), se han ido publicando trabajos que iban exponiendo los avances de la investigación en todas las disciplinas implicadas en el estudio de las ciudades. Resulta significativo que, coincidiendo con el apogeo de los estudios urbanos, se elaborasen y publicasen los primeros análisis sobre la dinámica y el estado de los estudios geográficos urbanos, tanto en otros países europeos (Dalmasso, 1980; Pinchemel, 1983, en el caso de Francia, p. ej.) como en España: Bosque Maurel (1983 y 1986), Carreras y Vilagrasa (1983), Carreras (1984), Gómez Mendoza (1989), Mas (1989), Santos Preciado (1992), etc.

Entre las últimas revisiones y, en consecuencia, con una amplitud temporal mayor, destacan los balances realizados por Tomé (2002) sobre la investigación en Geografía urbana histórica y por Capel (2003) y Morales y Méndez (2012) sobre las redes de ciudades y los sistemas urbanos. Más tarde han abordado la evolución de la Geografía urbana española y su reciente renovación Precedo (2010) y Rubio (2011). Una de las últimas revisiones, publicada por la Asociación de Geógrafos Españoles en el marco de un detallado análisis general de la investigación geográfica en España, hace acopio, con un enfoque crítico e interpretativo, de los trabajos en Geografía urbana de la última década del siglo xx y la primera del actual (González y Rullán, 2013).

\section{LAS MIRADAS DE LA GEOGRAFÍA SOBRE LAS CIUDADES DURANTE EL SIGLO XX}

La Geografía urbana se ha desarrollado relativamente tarde dentro de nuestro marco académico. Pese a la curiosidad de algunos geógrafos por la ciudad antigua (Ratzel y el propio Vidal de La Blache), hasta 1911, año en que Raoul Blanchard publicó su monografía sobre Grenoble, convertida en paradigma europeo de los estudios urbanos durante la primera mitad del siglo $\mathrm{xx}$, no puede decirse con propiedad que el fenómeno urbano constituyese un eje de atención prioritario para la Geografía, que llegará con cierto retraso frente a las interpretaciones 
del fenómeno urbano por otras disciplinas territoriales como el urbanismo y la arquitectura (Cerdá, Howard, p. ej.).

La preocupación por la ciudad, y en particular por el crecimiento del espacio urbano, se inicia a finales del siglo XIX, siendo una de las aportaciones pioneras la del norteamericano Adna Weber (1899), una aproximación a una perspectiva de conjunto del fenómeno urbano a partir del papel estructurador de los transportes. Pero es en los primeros decenios del siglo xx cuando comienza a hacerse evidente que las ciudades habían afianzado su papel como los principales marcos espaciales de la vida humana ya que el conjunto de la sociedad se estaba urbanizando rápidamente en los países más avanzados. Estos hechos incontrovertibles impulsaron el aumento de los estudios acerca de la ciudad, convertida en un objeto cada vez más pluridisciplinar.

Algunos autores sostienen que la Geografía urbana irrumpe a principios del siglo XX en un contexto en que las ciencias humanas tenían dificultades para captar la ciudad en su conjunto por lo que, en muchos sentidos, «se puede considerar la geografía urbana como la primera (disciplina) que propone e ilustra una aproximación a la ciudad, la «ciencia de las ciudades»»» (Berdoulay y Soubeyran 2010:42).

En Europa, las primeras obras explícitas de Geografía urbana, las alemanas (Schlüter, Hassert) y las francesas (Blanchard), íntimamente relacionadas con el paradigma regionalista y posibilista de la escuela vidaliana (Berdoulay, 2001), aportaron una metodología inductiva basada en tres elementos de análisis que han continuado siendo válidos durante mucho tiempo: la relación entre los factores naturales y el origen y desarrollo de las ciudades (emplazamiento y situación), el proceso de configuración de la realidad urbana desde una perspectiva histórica, su morfogénesis (evolución espacial) y, en tercer lugar, las actividades que sirven de base económica sobre la que se apoya la vida de la ciudad y sus habitantes (las funciones urbanas). La Geografía urbana clásica dirigió también miradas complementarias hacia los paisajes urbanos, las formas y los aspectos arquitectónicos, el análisis demográfico de la población urbana y sus relaciones con la región de la que forma parte la ciudad.

Este paradigma encontró su expresión más acabada en las investigaciones de los geógrafos franceses seguidores de Vidal de La Blache que, desde principios del siglo xx, realizaron importantes esfuerzos para pensar la ciudad como conjunto, en particular a través de las obras de algunos de su principales discípulos, como es el caso de Raoul Blanchard sobre Grenoble (1911) y Annecy (1916), Jacques Levainville sobre Rouen (1913) y Camille Vallaux sobre Péronne (1919), quien añadió un enfoque prospectivo que avanza una orientación planificadora, apoyada en el análisis empírico, con el fin de conseguir un desarrollo económico sostenido.

Desde una perspectiva más sociológica, fue Patrick Geddes (1915), considerado como el discípulo escocés de Élisée Reclus, quien aportó al estudio de 
las ciudades un nuevo lenguaje impregnado de naturalismo y de organicismo; el geógrafo Franz Schrader, pariente de Reclus, recogió las ideas de Geddes en Annales de Géographie de 1917.

La vinculación de la Geografía, la Historia y el Urbanismo en torno a la ciudad fue casi inmediata y se produjo a través de la revista La Vie Urbaine, que empezó a publicarse en 1919 por el Instituto de Urbanismo de la Universidad de París dirigida por el historiador Marcel Poëte, y en la que colaboraron desde el principio historiadores, historiadores del arte, geógrafos, arquitectos y urbanistas. La Vie Urbaine es considerada como la referencia de la ciencia urbanística en Francia, incluso con posterioridad a la publicación de Urbanisme en 1932.

Fue precisamente en La Vie Urbaine donde vio la luz en 1922 el artículo en el que Blanchard exponía su propuesta de «Un método de geografía urbana» dedicado a diseñar el análisis del espacio urbano en un momento en que el Urbanismo, como «ciencia de la ciudad», no establecía todavía diferencias entre la investigación fundamental y la acción o intervención práctica sobre el espacio urbano. Raoul Blanchard desarrolló y aplicó conceptos y métodos propiamente geográficos, diferentes de los históricos pero con un importante contenido histórico, utilizando un esquema cuyos puntos clave eran la situación, el emplazamiento, la evolución histórica, las funciones y la morfología. A partir de la segunda década del siglo el método de trabajo propuesto por Blanchard fue valorado como referencia indiscutible para el estudio científico de la ciudad, considerada, desde entonces, como objeto de análisis por sí misma, con un pionero enfoque integral y ecológico que pronto adquirió un carácter magistral tanto para los geógrafos como para los primeros urbanistas franceses. Como es el caso del arquitecto Léon Jaussely, primer presidente de la Sociedad de Urbanistas Franceses, fundada en 1924, quien afirmaba que «los geógrafos son los verdaderos padres del urbanismo» (Bielza, 2011).

La utilidad de la Geografía urbana para los urbanistas, en proceso de profesionalización, fue percibida con claridad por Jaussely, como se observa en el anteproyecto de plan para Barcelona que presentó al concurso internacional convocado en 1903 y con el que obtuvo el primer premio. El proyecto definitivo, aceptado por el Ayuntamiento en 1907, ofrecía una visión global del conjunto urbano (vías de comunicación, equipamientos públicos, parques y zonas comerciales, industriales y residenciales), a diferencia de las otras propuestas. Berdoulay y Soubeyran (2002) consideran que este plan contiene un proyecto estratégico de desarrollo productivo de la ciudad a través de la racionalización de su funcionamiento y que Jaussely fue el urbanista más sensible a la contribución de los geógrafos vidalianos franceses ${ }^{1}$.

1. Berdoulay y Soubeyran, «Nota de la Traductora», Josefina Gómez Mendoza. Tanto Blanchard como Geddes apuntaron precozmente la relación de la Geografía Urbana y el planeamiento y la notable aportación que la primera podía hacer a la ordenación del espacio urbano. 
En el artículo de Blanchard quedaba ya prefijada la secuencia del «relato» del análisis urbano y los aspectos esenciales de la estructura de los estudios sobre la ciudad, identificados en general con el conocimiento de la evolución histórica de la ciudad: la morfología (el aspecto), las funciones (los papeles) y el funcionamiento interno de la ciudad (la vida urbana y de sus habitantes).

Morfología (plano, tejido viario, tipologías edificatorias, etc.), funciones y estructura social de las ciudades van a ser tres aspectos complementarios, que constituyen una constante como ejes centrales de los estudios de Geografía urbana desde su fase germinal hasta la actualidad, en distinto orden de prioridades según el predominio de los enfoques espacial, económico o social. Aspectos que, por otra parte, coinciden en gran medida con los objetos de estudio de la Historia puesto que tienen su propia concreción en cada etapa y modelo histórico de ciudad. E, igualmente, son temas de trabajo comunes con la Historia del Arte, la Arquitectura y el Urbanismo.

Tanto los geógrafos (Halbwachs) como los historiadores urbanistas (Pierre Lavedan o Marcel Poëte) dieron primacía de inmediato a los aspectos morfológicos, que constituyeron una significativa aportación a la primitiva Geografía urbana francesa. Muchos años más tarde, el geógrafo J. Tricart (1954) consideraba la morfología urbana como el punto de convergencia del urbanismo, la sociología, la historia, la economía política o el derecho. Asimismo, el arquitecto Aldo Rossi concibe la ciudad como una construcción en el tiempo, una estructura cuyo significado cambia a lo largo de la historia; su estudio sobre la arquitectura de la ciudad (1981) ha sido una de las obras de referencia sobre la morfología urbana.

La morfología urbana, asimilada habitualmente al paisaje, constituyó el enfoque preferente de la primitiva Geografía urbana (Hassinger, Passarge, Bobek, la escuela de Viena, Toschi) que prolongó sus aportaciones hasta mediados del siglo xx. Cabe destacar en este contexto los estudios del ingeniero alemán O. Jürgens y del geógrafo $O$. Jessen sobre las ciudades y los paisajes urbanos españoles respectivamente (Bielza, 2011).

Los estudios de las funciones urbanas, identificadas con las actividades económicas, tuvieron como punto de partida las teorías de la localización, formuladas por economistas y geógrafos desde comienzos del siglo XIX (J.H. von Thünen) y de la primera mitad del siglo xx (Weber, Christaller, Lösch).

El análisis de la sociedad urbana se introdujo más tarde en los trabajos geográficos por influencia de la escuela de ecología humana de Chicago (Robert E. Park) si bien los estudios urbanos de J. Tricart y P. George contenían también un notable enfoque social.

Viene lo anterior a avalar la aserción de que la Geografía urbana ha sido, desde sus orígenes, «una disciplina plural que ha tratado de articular los aspectos morfológicos, funcionales y sociales de la ciudad dentro de una variedad de 
enfoques y metodologías» (Juaristi, 2015:233). Porque la Geografía urbana no ha sido una ciencia estática, sino que la temática objeto de estudio y los métodos de investigación han ido variando y ampliando sus horizontes desde sus comienzos al compás del desarrollo de nuevos paradigmas y de las metodologías inherentes a ellos, así como por la influencia ejercidas por otras disciplinas interesadas también en el tratamiento del espacio urbano.

Los primeros estudios geográficos sobre ciudades españolas bajo un enfoque estrictamente espacial se desarrollaron relativamente tarde; fueron elaborados en los años treinta del siglo Xx y continuados tras la finalización de la Guerra Civil, siguiendo el paradigma y la metodología de la Geografía francesa de las primeras décadas del siglo.

A finales de los años 1920 se había publicado la primera monografía urbana moderna, la del historiador Carles Rahola sobre Girona (1929) al tiempo que Pau Vila, en su Resum de Geografia de Catalunya (1928-35), establecía las bases de los estudios de Geografía urbana a partir también del esquema metodológico de Blanchard. En 1932 se publicó el primer estudio monográfico sobre Madrid (Guinart y Mombeig) y en 1934-1936 otros sobre Barcelona elaborados por el geógrafo e historiador hispanista francés Pierre Vilar; pero en los dos casos se trataba de artículos publicados en revistas francesas.

El verdadero introductor de los análisis geográficos urbanos en España fue Manuel de Terán, que realizó una estancia en París, pensionado por la Junta para Ampliación de Estudios, en el Institut de Géographie y en el Institut d'Urbanisme (Gómez Mendoza, 2004). Allí entró en contacto con Poëte y conoció los artículos publicados en La Vie Urbaine, que le permitieron aprehender el método de Blanchard que aplicó a sus estudios sobre algunas ciudades andaluzas (1936), Calatayud, Daroca y Albarracín en 1942, Sigüenza (1946) y Toledo. En este marco, aunque algo más tarde, se inscriben sus obras sobre Madrid (1961) consideradas como un modelo de trabajo para la Geografía histórica de las ciudades y el urbanismo (Terán y Marías, 2004).

En los trabajos de Manuel de Terán y su coetáneo Amando Melón quedaron apuntadas las principales líneas temáticas de la Geografía urbana clásica española: el crecimiento de las ciudades, los modos de apropiación y uso del suelo urbano, las inversiones inmobiliarias y la estructura de la propiedad, la forma y organización del plano y, en definitiva, los elementos estructurales de la ciudad, entendida como un espacio social, funcional y morfológicamente diferenciado y segregado.

A partir de estas obras pioneras, a finales de los años cincuenta y principios de los sesenta se realizaron algunas monografías locales bajo una perspectiva temporal y morfogenética con el objetivo de reconstruir la evolución histórica de los espacios urbanos, la mayor parte de las cuales correspondían a tesis de licenciatura (tesinas) y tesis doctorales. Una de las primeras que vio la luz, en 
1962, fue el estudio que hizo sobre Granada el profesor Joaquín Bosque Maurel, una obra que ha sido considerada como un hito y reeditada en edición facsímil (1988).

En el último tercio del siglo xx, el conocimiento de los espacios urbanos se ha convertido en una de las preocupaciones prioritarias para la Geografía tras la culminación del proceso de urbanización (de Vries) que ha convertido a la ciudad en la forma dominante de asentamiento y hábitat humano y, en consecuencia, en el fundamento de la organización territorial. Este proceso ha tenido lugar de forma simultánea a la emergencia de notables mutaciones epistemológicas en Geografía y al desarrollo de nuevas corrientes conceptuales y metodológicas en las que el papel de la Geografía urbana ha sido fundamental, hasta el punto de que se puede afirmar, sin exageración, que desde la década de los años 1960 la Geografía urbana ha llegado a ser una rama esencial de la Geografía.

Lo mismo sucede en España, pero con una cierta demora. Coincidiendo con la etapa en que la urbanización se empezó a percibir como el proceso de transformación territorial más notable y las ciudades como los asentamientos predominantes, los espacios urbanos fueron entendidos como uno de los objetos fundamentales de estudio para la Geografía española. En correspondencia, a partir de este período alcanzaron una gran importancia los estudios que versaban sobre los procesos de urbanización (Juaristi y Vinuesa y Vidal, entre otros).

No puede sorprender, en consecuencia, que la Geografía urbana española alcanzase su mayor expansión a partir de los años 1970, hasta el punto de que varios autores consideran que ésta fue la rama que se convirtió en el principal motor de la disciplina y contribuyó en mayor medida a la modernización de la Geografía y a su configuración como disciplina científica de gran impacto social y económico (Precedo, 2010). Así, los setenta fueron los años en que se produjo una importante ampliación del ámbito espacial de estudio de la Geografía urbana y, por otro lado, una gran renovación epistemológica y metodológica. Fue ésta, como algunos autores prefieren afirmar, la época «dorada» de la Geografía urbana española (García Ballesteros), en coincidencia, no por casualidad, con el proceso de democratización política y la demanda de conocimiento riguroso de los espacios urbanos, como base de las intervenciones urbanísticas que empezaron a sacar las investigaciones geográficas del ámbito académico.

Desde unos años antes, y hasta finales de los años setenta, la elaboración de numerosas memorias de licenciatura y tesis doctorales sobre distintos espacios urbanos españoles dio origen al afianzamiento de lo que podemos considerar como la primera gran generación de geógrafos urbanos españoles. En ella cabe integrar también a los geógrafos y geógrafas que estudiaron los espacios urbanos desde numerosas universidades, entre ellas las de Barcelona, Madrid, Oviedo, Santiago, Sevilla, Valladolid, Zaragoza, etc. La extraordinaria pujanza de la Geografía urbana de esos años se materializó en la publicación de obras 
emblemáticas, como el estudio sistemático de las ciudades españolas en siglo XIX que realizó Quirós Linares (1991, 2009) y que puede considerarse como el ápice de la Geografía urbana histórica.

Sin embargo, la mayor parte de los estudios urbanos españoles tuvieron todavía un carácter monográfico y consistieron en el análisis de la evolución temporal y el contexto regional de una ciudad concreta, o de una parte de ella, a partir de tres elementos fundamentales: el emplazamiento y la situación, la dinámica económica, social y espacial en clave histórica y los aspectos fisionómicos relativos a la morfología y los paisajes urbanos. En la mayoría de los casos, también, estos trabajos presentaban una notable convergencia entre la Geografía y la Historia, lo que resulta lógico habida cuenta de que la práctica totalidad de los autores tenían formación académica histórica. Algunos autores han calificado, con gran acierto, estas monografías sobre ciudades como estudios «biográficos» que proporcionan las claves de la interpretación del espacio urbano a través de los procesos de su formación (Ocaña, 1997). Sólo en contadas ocasiones se transitó desde las monografías empíricas a los estudios teóricos y de conjunto a la búsqueda de tipologías, siguiendo, con cierto retraso, los planteamientos críticos de Dickinson y del Symposium de la Unión Geográfica Internacional sobre Geografía Urbana, celebrado en Lund en 1962, y las propuestas funcionales de la Nueva Geografía.

En este contexto hay que entender la creación y el funcionamiento de organismos como el Laboratorio de Urbanismo de la E.T.S.A.B. que, aglutinado en torno a Manuel de Sola-Morales i Rubió, asumió el empeño de dar a conocer las nuevas tendencias urbanísticas mundiales. Asimismo, el Instituto de Estudios Urbanos de la Universidad Complutense que organizó en mayo de 1987 las Primeras Jornadas de Estudios Urbanos con el fin de presentar una especie de catálogo de algunas de sus futuras líneas de investigación: los problemas del medio ambiente urbano, la dimensión histórica de las ciudades y las políticas de protección del patrimonio histórico arquitectónico de nuestras ciudades, los comportamientos de la población urbana, el impacto en las ciudades de los cambios sociales y económicos, entre otras (García Ballesteros). O el Grup d'Estudis Urbans, coordinado por Joan Vilagrasa i Ibarz en la Universidad de Lleida, organizador de las reuniones científicas denominadas Setmana d'Estudis Urbans y celebradas en dicha ciudad durante varios años.

Como antes apunté, y en relación con las inquietudes de numerosos geógrafos urbanos, también es la etapa en que se produjo un profundo cuestionamiento de los métodos de análisis clásicos y la llegada de nuevos paradigmas, algunos de ellos aparentemente incompatibles entre sí, todo lo cual condujo hacia la ruptura de la relativa unidad epistemológica preexistente y, hasta cierto punto, a la marginación de la Geografía urbana clásica, acusada de tener un enfoque historicista y tradicional, más descriptivo que explicativo, y, por ello, poco científico 
y escasamente eficaz para dar respuesta a las demandas sociales, en particular a los requerimientos del planeamiento urbano.

Por esos años se incorporaron los enfoques behavioristas sobre la imagen mental y la percepción de la ciudad (Bailly, Yi-Fu Tuan) por influencia de los arquitectos urbanistas (Lynch) y los sociólogos (Ledrut). A los anteriores hay que unir la influencia de las aportaciones de las teorías sobre la ocupación del suelo y el crecimiento urbano de la escuela de ecología urbana de Chicago, con precedentes en Barrows y A. Hawley.

Otra corriente emergente fue la interpretación de la ciudad en su territorio aplicada ya por Casassas (1976) para Barcelona, una de las aportaciones más notables de la Geografía urbana, poniendo el acento en el estudio de las relaciones entre la ciudad y el espacio regional, la estructura y la jerarquía funcional, el área funcional o de influencia de las ciudades en el territorio circundante y la clasificación funcional de la ciudades.

Pero, sobre todo, se consolidó la concepción sistémica que venía a reforzar, con una perspectiva más integradora, desde un punto de vista dinámico y con una clara vocación hacia el planeamiento urbanístico, los enfoques morfológicos y estructurales clásicos del espacio urbano (Bastié y Dezert, Bourne, Carter). Entre los introductores de este paradigma en España es obligado citar a H. Capel (1974) y E. Murcia (1979).

A través de esta nueva conceptualización territorial, la Geografía urbana se articuló en torno al estudio del sistema urbano desde un punto de vista teórico y empírico y bajo dos perspectivas prioritarias: el sistema intraurbano y el sistema interurbano, al que se asignó un papel decisivo en el proceso de desarrollo económico a partir de la teoría sobre los procesos de difusión elaborada por Hägerstrand y Brown. De esta forma, también en España se introdujeron y aplicaron nuevos conceptos como el de red urbana y sistema urbano. Estos enfoques, funcional y sistémico, facilitaron la convergencia temática y metodológica de geógrafos, economistas y arquitectos, aunque con objetivos diferentes, y la conexión con la planificación territorial y el urbanismo, en tanto en cuanto el sistema urbano se consideró como el pilar esencial de los planes regionales y locales.

La Geografía urbana, al igual que las demás ciencias sociales, asumió también la corriente teorético-cuantitativa del neopositivismo por influencia de los geógrafos anglosajones (Bunge, Chorley, Haggett, Harvey, Schaefer), a la búsqueda de un cuerpo de doctrina coherente y válido tanto para las ciencias naturales como para las sociales. El neopositivismo geográfico aportó un lenguaje neutral, estadístico-matemático, y la modelización de los procesos para expresar la correspondencia existente entre el mundo teórico y el real (Santos, 1992). El resultado fue la producción de estudios basados en la interpretación estructural de los usos del espacio urbano a partir de la distribución de actividades y 
clases sociales, poniendo el énfasis en los mecanismos que fraguan la realidad urbana. Se trataba más de "captar la estructura del objeto y no su esencia», como afirmaban Capel y Urteaga en 1981. Si bien, es preciso reconocer que, en el caso de la Geografía urbana española, la asimilación de esta corriente no fue especialmente nutrida, el resultado fue la producción de estudios basados en la interpretación estructural de los usos del espacio urbano, poniendo el énfasis en los mecanismos económicos que fraguan la realidad urbana. En este marco puede inscribirse, aunque sea de forma parcial, la atención preferente que se otorgó durante un tiempo a la relación entre el espacio urbano y la actividad industrial (Congreso de la Asociación de Geógrafos Españoles de 1975 con el lema Ciudad e Industria, 1977).

Asimismo, el enfoque social se intensificó a partir de la influencia de obras como las de M. Castells², H. Lefebvre, Herbert, Folin, Johnston, Knox o Ley, entre otros, hasta el punto de derivar en una geografía radical (Wolch, 2003) comprometida y vinculada a las teorías críticas de origen marxista (Precedo, 2010; Juaristi, 2015). Una de las aportaciones esenciales al nuevo paradigma radical marxista fue la obra de D. Harvey (1977) y, más tarde, el trabajo de Milton Santos (1990), quien enlaza el materialismo con una visión historicista de la producción social del espacio (Gintrac, 2013).

El espacio, en general, y el urbano, en particular, es concebido como un «producto social» (Lefebvre) resultante de la contraposición de intereses de los grupos sociales de los diferentes tipo de sociedad que lo utilizan y, por consiguiente, refleja espacialmente la organización social en toda su complejidad y relaciones. Por ello, el espacio urbano es analizado como un subsistema socioespacial que forma parte de un sistema territorial más amplio; su estudio pone el acento en el análisis del uso social de la ciudad, las pautas de comportamiento social de sus habitantes y los procesos y mecanismos del crecimiento urbano y de la transformación morfológica que originan (I. Solà-Morales).

No resulta sorprendente que sea en estos años cuando se publicase el texto que puede ser considerado como la primera obra general de la Geografía urbana española, el estudio de Horacio Capel (1975), Capitalismo y morfología urbana en España, que establece y explica la secuencia del proceso evolutivo de las ciudades españolas a través de la relación entre las formas y los mecanismos que las han originado, los procesos de producción del espacio y los agentes que han intervenido en tales procesos. El propio H. Capel (2010) afirmaba años después que «La renovación de un marxismo crítico en los años 1960 fue un acontecimiento de gran repercusión en el campo de los estudios urbanos. En este sentido Henri Lefebvre fue uno de los primeros que lo hizo de una forma convincente».

2. «el proceso de formación de las ciudades está en la base de las redes urbanas y condiciona la organización social del espacio» (Castells, 1971:75). 
El proceso de actualización y diversificación, someramente reseñado, no redundó, no obstante, en la desaparición del legado epistemológico y metodológico previo sino que, bien al contrario, fortaleció la Geografía urbana clásica, que adoptó métodos y temas de mayor rigor científico y preocupación social (Campesino, 1985). De hecho, durante este tiempo siguieron teniendo una extraordinaria presencia las aproximaciones locales, de carácter integral, a los espacios urbanos, analizados desde una perspectiva evolutiva histórica. Pero, a la vez, la ampliación del proceso de urbanización, la aceleración de la dinámica de crecimiento de los núcleos urbanos y el aumento de su complejidad interna y de las interrelaciones externas, estimularon la selección de objetos de estudio más abarcables, tanto por sus dimensiones como por la temática, lo que se tradujo en la proliferación de estudios de partes de la ciudad (barrios periféricos, centros urbanos y cascos históricos, áreas de Ensanche, etc.), con una escala de análisis más accesible.

Varios son los aspectos que alcanzaron primacía desde un punto de vista temático. Entre ellos destaca el estudio del papel de los agentes sociales en la generación de suelo urbano, del precio del suelo (García Ballesteros y Redondo, Mas) y de la estructura de la propiedad del mismo (García-Bellido) así como la promoción inmobiliaria (García-Bellido y González, Mas). Entendidos como los instrumentos de la producción social del espacio urbano y de la morfogénesis urbana, pronto se convirtieron en los temas estelares de la Geohistoria urbana española apoyados en la explotación de nuevas fuentes fiscales (Carreras, Mas, Pillet, Tatjer, Vilagrasa, etc.) y con especial predilección por el análisis de los procesos durante el siglo XIX, en especial su segunda mitad y el primer tercio del siglo xx, el período de la denominada «transición urbana». Tales investigaciones otorgaron especial protagonismo al estudio de los Ensanches de población a partir de la morfología catastral histórica. Entre los trabajos que responden a esas características cabe citar también el de geógrafo hispanista francés Coudroy de Lille sobre los Ensanches españoles (1996) y, tres años después, el trabajo colectivo sobre el mismo asunto coordinado por López Trigal (1999).

Igualmente llegaron a ser temas de investigación preferentes la relación de la ciudad con las actividades económicas, la incidencia en el trazado urbano de las infraestructuras de transporte y los elementos estructurantes en general, los modelos de ocupación del suelo, las formas de construcción y la tipología de edificios y viviendas, etc. Una línea de investigación que se consolida entonces y se prolonga durante los años noventa, e incluso más, es el estudio de la vivienda y los barrios obreros y sus efectos sobre la ampliación del espacio urbano entre mediados del siglo xIx y el primer tercio del xx (Castrillo, Díez de Baldeón, Oyón, Tatjer).

Otro aspecto que empezó a atraer el interés de la investigación geográfica fueron los centros urbanos y los cascos históricos, lo que incentivó la formación 
de equipos pluridisciplinares de geógrafos, historiadores y arquitectos y permitió la renovación de las perspectivas morfológica, historicista y paisajística (Carter, Whitehand). En relación con lo anterior, aunque con enfoques parcialmente diferentes, tuvo lugar también la continuación y consolidación del tratamiento morfológico de los espacios urbanos.

\section{LA GEOGRAFÍA URBANA ESPAÑOLA A PRINCIPIOS DEL SIGLO XXI}

Los espacios urbanos han experimentado grandes cambios desde los años noventa del siglo xx, sobre todo a causa de los procesos vinculados a la globalización, la nueva forma de colonización mundial. Las transformaciones acaecidas en todo tipo de países, sobre todo en los avanzados y emergentes, y las nuevas relaciones entre los procesos globales/mundiales y los locales han impulsado, asimismo, significativas modificaciones en los estudios urbanos desde las últimas décadas del siglo xx.

La globalización económica ha contribuido a reforzar el papel de las ciudades en el sistema económico mundial (Sassen, Castells, Hall), lo que ha inducido el desarrollo de una temática orientada a estudiar la dimensión global de las ciudades bajo el enfoque de la competencia y competitividad urbanas y en marcos territoriales más amplios (Bosque Maurel, Claval, Smith). Anthony D. King, en su obra Global cities (1990), exponía que el estudio de las ciudades en términos de sistemas cerrados, estatales o regionales, había sido sustituido por una perspectiva global y mundial (Zumín, 1994). Al mismo tiempo que, como reacción compensatoria, se ha ido revalorizando el lugar y, por ende, el conocimiento de la dinámica urbana local. Todo lo cual, de una u otra forma, ha redundado en una revitalización y renovación del interés por la temática urbana. En resumen, se puede afirmar que los estudios urbanos están adoptando un enfoque «glocal», priorizando, en unos casos, la dimensión global y, en otros, la local, moviéndose entre análisis teorizantes, generales y abstractos, en unas ocasiones, y estudios empíricos y localistas, meramente descriptivos, en otras (Borja y Muxí, 2004).

Por otro lado, las ciudades se han ido haciendo cada vez más complejas, no sólo por sus dimensiones sino también por su forma, sus estructuras y por los modos de relación de las nuevas jerarquías espaciales. La definición de la ciudad que daba Mumford en 1961 como «la forma de una relación social integrada con centro y límites, cuyas capas revelan la síntesis de sus diferentes épocas históricas», ha quedado obsoleta ante la consolidación de nuevos modelos de ciudades. Así, los tipos de espacios urbanos emergentes, las aglomeraciones urbanas, tienen una nueva estructura física y una forma policéntrica a partir de numerosos núcleos interconectados (I. Solà-Morales) que configuran una ciudad flujo, compleja y desordenada, cuyo centro no es un lugar sino el tiempo (García y Segado). 
En relación con los cambios mencionados, la Geografía urbana española ha ido evolucionando desde los años noventa hasta la actualidad en varias direcciones. Por un lado, se ha producido una notable ampliación del número de investigadores que trabajan en este campo de estudio. Por otro, pero dependiendo también de lo anterior, se han diversificado todavía más las temáticas abordadas, se ha reforzado el acercamiento al urbanismo y se ha producido un relativo distanciamiento respecto a la Historia. Por lo que se refiere a este último aspecto, uno de los factores decisivos ha sido la separación de las disciplinas de Geografía e Historia en diferentes Licenciaturas, primero, y Grados, después, con escasa conexión de contenidos y métodos entre sí, pese a impartirse por regla general en las mismas Facultades universitarias..

No obstante, la valoración del patrimonio y el paisaje urbano se está convirtiendo en un nuevo punto de encuentro de la Geografía y la Historia urbanas que, a medio plazo, podría redundar en el reforzamiento de las investigaciones centradas en el análisis de los elementos heredados subsistentes, en el origen histórico de las formas y los paisajes urbanos presentes y en las propuestas para su integración en la estructura y la morfología urbana actuales y su preservación como recursos vinculados a las actividades turísticas.

El análisis de los paisajes urbanos, un campo de trabajo transversal donde los haya, ha atraído la atención de numerosos geógrafos, en tanto que objeto de estudio en sí mismo y como instrumento de planificación, en el entorno temporal de la publicación de la Convención Europea del Paisaje en el umbral del siglo (Florencia, 2000), con el objetivo de ordenarlos, protegerlos y gestionarlos.

La temática del paisaje urbano y la imagen visual de la ciudad, que contaba ya con importantes precedentes, lejanos y próximos, en Europa (Sitte, Cullen, Lynch, Rimbart) y en España, es abordada hoy por numerosos autores que tratan la historia de la ciudad y el paisaje urbano desde diferentes perspectivas, sobre todo el paisaje urbano histórico como recurso turístico, pero también como paisajes generados por los procesos de urbanización actuales y de los efectos de las «burbujas» y las crisis inmobiliarias, o tomando en consideración la integración de la naturaleza en la ciudad como forma de regeneración y la construcción de los paisajes urbanos. Entre otros, un geógrafo, F. Zoido, que en la actualidad dirige el Centro de Estudios Paisaje y Territorio de Andalucía, ha sido uno de los principales estudiosos y teorizante de los paisajes urbanos y de su integración en la planificación territorial y urbanística a través de aportaciones en las que sistematiza el estudio del paisaje urbano diferenciado del enfoque morfológico clásico (Zoido, 2012).

En concordancia con lo anterior, cabe destacar la emergencia y renovación de las investigaciones relacionadas con el patrimonio urbano, no sólo el monumental y artístico de las ciudades patrimoniales, sino también el patrimonio industrial o conexo con las antiguas infraestructuras de transporte, etc. 
En el mismo sentido, es preciso resaltar que últimamente se está produciendo un leve resurgimiento del interés por la Geografía urbana histórica y los enfoques morfogenéticos, en España al igual que en otros países europeos (Whitehand), porque los paisajes urbanos tienen una estrecha relación con las estructuras morfológicas de la ciudad, aunque no sean lo mismo. Así, la vinculación entre morfología y paisaje urbano ha proseguido, revitalizada, a través de valiosas aportaciones como la obra de H. Capel (2002) sobre la Morfología urbana I. Sociedad, cultura y paisaje, donde considera éste como un reflejo de la cultura, de la economía y de las funciones urbanas. Se sigue trabajando activamente sobre la interpretación de los cambios estructurales y morfológicos (Conzen), si bien, el interés se ha desplazado desde los estudios clásicos del espacio urbano completo, o de determinados barrios de la ciudad consolidada, hacia las nuevas áreas suburbanas y periurbanas (residenciales, de servicios, tecnológicas, industriales, etc.) como ejemplos de la nueva forma difusa de la ciudad y de suburban sprawl.

La materialización más acabada de la forma urbana tradicional son los cascos históricos, sobre los que ha vuelto, renovada, la mirada de la Geografía urbana. La preocupación por los cascos y centros históricos, y su recuperación como seña de identidad de nuestras ciudades en el marco de la globalización frente a las formas y tipologías estandarizadas de los espacios suburbanos y periurbanos contemporáneos, arranca de los años noventa pero se intensifica en la primera década del siglo actual. Otro factor de estímulo de este campo de investigación ha sido la búsqueda de respuestas a la demanda social y económica de la restauración de los centros históricos, incluidas las áreas de ampliación decimonónica (ensanches burgueses y barriadas obreras de mayor calidad) como espacios atractivos para la inversión en nuevos usos terciarios (servicios financieros, profesionales, de ocio y turismo) que pueden proporcionar una mejora de la calidad de vida de los ciudadanos.

Pero, además de los temas constantes y recurrentes, los objetos de investigación de las y los geógrafos urbanos, como es lógico y había ocurrido antes, se han ido adaptando a las transformaciones espaciales, socioeconómicas y culturales contemporáneas e, igualmente, los objetivos científicos perseguidos (González y Rullán, 2013). Una escrupulosa revisión de las publicaciones desde la última década del siglo pasado hasta el momento actual nos proporciona un significativo muestrario de la extraordinaria diversificación temática y metodológica que caracteriza hoy la Geografía urbana española.

En términos generales, se mantienen las dos líneas principales que han sido una constante en la Geografía urbana desde hace más de medio siglo: el análisis interno, en tanto que estudio del espacio interior de la ciudad, y el análisis externo, que aborda cada vez con más frecuencia el estudio de las ciudades en el territorio, las redes y los sistemas urbanos. Otra temática que se prolonga 
desde la etapa anterior es el estudio de los procesos de urbanización en sus formas pasadas y presentes y, en menor medida, la interpretación general y la caracterización de los espacios urbanos.

Junto a los anteriores, destacan otros como objetos de análisis específicos emergentes.

Así, se han dedicado numerosos trabajos a explicar las mutaciones de los sistemas urbanos a diversas escalas, desde las ciudades pequeñas y medias hasta la configuración de las aglomeraciones urbanas, con especial hincapié en los procesos de dispersión y metropolitanización (Feria, Nello).

Las repercusiones de la construcción de las infraestructuras de transporte sobre los espacios y los sistemas urbanos, en particular las vías y líneas de gran velocidad y altas prestaciones y los aeropuertos, han atraído el interés de muchos investigadores poniendo el acento en las mutaciones inducidas en las estructuras espaciales y en la modificación de las jerarquías urbanas (Alonso y Bellet). Igualmente, está siendo un tema de notable preocupación geográfica el análisis de las mutaciones morfológicas y funcionales relacionadas con el impacto de dotaciones y equipamientos y, sobre todo, de nuevas actividades económicas, en particular con la incidencia del turismo (Blázquez, Rullán). Así como los efectos sobre la ciudad de los cambios sociales, sobre todo los relacionados con la inmigración extranjera y los procesos de vulnerabilidad, exclusión y segregación socioespacial, gentrificación, etc.

Por otra parte, la necesidad de dar respuesta a nuevas demandas sociales y territoriales ha impulsado la orientación hacia el análisis de las políticas y las prácticas de planificación territorial, estratégica y urbanística, y sectorial y la aplicación de sus resultados al desarrollo urbano, la promoción y el marketing de las ciudades así como a la sostenibilidad ambiental urbana (Gaja, Indovina), lo que, como señalaba antes, ha acercado más la Geografía urbana al Urbanismo, entendido como práctica política y espacial, y ha impulsado la intervención más intensa de los geógrafos en la planificación urbanística. Así, el planeamiento se ha convertido en un objeto de estudio consolidado, tanto se trate del planeamiento urbanístico general como de los planes especiales y parciales, con singular interés en los planes de rehabilitación de los cascos históricos y en los planes de intervención en los «desarrollos periféricos» como formas recientes de producción de suelo urbano y, en definitiva, de construcción de la ciudad. Igualmente, son estudiados los efectos sobre el espacio urbano de los planes turísticos, de vivienda, de transportes y, en general, de la planificación estratégica, con objetivos interpretativos y propositivos.

Son abundantes también los estudios, generalmente críticos, sobre las consecuencias de las políticas de suelo y vivienda, la evolución del sector inmobiliario y las secuelas de la especulación urbanística y la artificialización del suelo en relación con la «burbuja» inmobiliaria (Burriel, 2008; Valenzuela, 2013), 
primero, su estallido posterior y la crisis del sector de la construcción en la actualidad, un asunto que ya había sido estudiado con anterioridad vinculado con el boom edificatoria de los años sesenta (Gormsen). En relación con lo anterior, se han abierto líneas de trabajo sobre el uso de los espacios públicos y los procesos de privatización de los mismos y, en general, del suelo urbano.

Si bien siguen primando los trabajos sobre la urbs, la dimensión arquitectónica y espacial, no se ignora que la ciudad es también una idea, un concepto, una percepción y una imagen por lo que han empezado a alcanzar importancia las investigaciones sobre la representación cartográfica, fotográfica, cinematográfica, literaria, pictórica, etc. de los espacios urbanos.

La dinámica espacial urbana ha sido abordada por incontables autores recurriendo de forma sistemática al uso de cartografía y planimetría, generalmente con la finalidad de ilustrar lo expuesto en los textos como resultado de la investigación realizada a partir de otras fuentes documentales. En bastantes menos ocasiones se han utilizado los mapas y planos históricos como fuentes por sí mismos para cimentar la investigación en la información obtenida directamente a partir de estos documentos. Sin embargo, en buena parte de los más recientes trabajos, la cartografía, histórica y actual, incluso digital, ha adquirido un protagonismo sobresaliente y no sólo con un carácter ilustrativo sino como fuente documental.

En relación con el recurso cada vez mayor a la cartografía histórica, es obligado hacer referencia a la elaboración de atlas urbanísticos históricos (da Costa) para un significativo número de ciudades españolas. Este tipo de obras, de gran tradición en otros países (Bocchi y Guidoni, para las ciudades italianas, Pinol, para las francesas, p.ej.), empezó a elaborarse en los años noventa para las principales urbes españolas (Barcelona y Madrid) y, de forma general, la obra pionera sobre las ciudades de la península ibérica de Guàrdia, Monclús y Oyón (1994 y 1996). Más adelante vieron la luz los atlas de otras ciudades como Valencia, Zaragoza, Sevilla, Valladolid, Cádiz, Lérida o Gerona; incluso atlas urbanos a escala regional como es el caso del Atlas Histórico de las ciudades gallegas durante los siglos XVII al XX (Cardesín).

La fotografía, histórica y reciente, ha pasado a ser otra fuente documental para el conocimiento de la morfología, tipología edificatoria y paisaje de los espacios urbanos una vez reconocido y aceptado su valor incontestable como instrumento de investigación, especialmente en el análisis de los procesos de cambios paisajísticos, a condición de que se disponga de series de imágenes que reflejen distintos momentos de la realidad urbana. Siguiendo la línea explorada en Francia por Mendibil, se está preconizando el uso de la imagen fotográfica como una eficaz herramienta de investigación (Canosa et alii). El recurso a la fotografía histórica «a pie de calle» se completa con el uso de la fotografía aérea 
y las imágenes de satélite utilizadas en igual medida por geógrafos urbanos y urbanistas.

El empleo de nuevos instrumentos y herramientas guarda estrecha relación con los cambios concernientes a los métodos utilizados que, como es natural, se ajustan a la nueva temática. En la mayoría de los casos se trata de métodos clásicos actualizados: explotación y elaboración de estadísticas, análisis crítico de la legislación, normativa y documentación del planeamiento y de la cartografía asociada a éste, el inexcusable trabajo de campo, una seña de identidad del trabajo geográfico, etc. Pero, cada vez en mayor medida, se han introducido nuevas herramientas y métodos a partir del diseño de indicadores, del uso y elaboración de planimetría y cartografía digital a través de Sistemas de Información Geográfica y del recurso de las nuevas Tecnologías de Información y Comunicación.

A partir de los años noventa, se ha producido una extraordinaria renovación tecnológica e instrumental. Si la utilización de la fotografía aérea, como fuente, y la fotointerpretación, como método, fueron esenciales antes para el estudio de los usos de suelo, desde finales del siglo xx las TIG (sistemas de información y teledetección) han abierto enormes posibilidades al análisis de los espacios urbanos. Por otro lado, las nuevas tecnologías multimedia han venido a enriquecer los instrumentos que geógrafos, urbanistas, historiadores, etc. utilizan para analizar la ciudad y para facilitar la aprehensión visual de su espacio urbano.

Pese a la notable renovación temática y metodológica reciente, no sería ecuánime concluir sin mencionar algunas limitaciones de la Geografía urbana española que han sido puestas de manifiesto en varias ocasiones. Entre otras, y sobre todo, el bajo nivel de reflexión teórica que muestra buena parte de los trabajos de investigación recientes. Bien es verdad que, concluida la «revolución» de los años setenta y ochenta, la mayoría de los autores no adscriben su trabajo a marcos teóricos o paradigmas rígidos sino que, haciendo gala de una mayor flexibilidad epistemológica, se apoyan en los aspectos más positivos y eficaces de diferentes paradigmas, lo que ha redundado en una cierta homogeneidad en los fundamentos teóricos utilizados para abordar la problemática urbana.

La escasa reflexión teórica y metodológica, así como la asimilación de contenidos y métodos procedentes de otras disciplinas, sin una crítica rigurosa, ha provocado una pérdida relativa del peso específico de la Geografía urbana en el contexto científico, lo que a juicio de algunos autores, está conduciendo a la «actual banalización de los estudios geográficos sobre la ciudad y la sociedad urbana» ya que «la aplicación de técnicas sin ideas que las soporten no dejan de ser otra cosa que una instrumentación operativa vacía de contenido» (Precedo, 2010).

Por otra parte, aunque en la mayoría de los casos el tratamiento de todos los aspectos mencionados, tanto tradicionales como nuevos, se hace de forma 
analítica y, en muchas ocasiones, desde un punto de vista crítico, no faltan tampoco los trabajos de ámbito local que todavía mantienen un carácter descriptivo al estudiar los procesos y dinámicas espaciales, poniendo la atención más en los efectos territoriales que en las causas que los engendran, de acuerdo con la antigua tradición geográfica de base inductiva (González y Rullán, 2013).

Como conclusión, se puede afirmar que la Geografía Urbana se encuentra hoy en una encrucijada a la búsqueda de nuevas formas de comprender el espacio urbano a partir de su encuentro con otras ciencias sociales abiertas hacia lo urbano (Hiernaux, 2006). Como afirmaba Milton Santos, la Geografía (urbana) se está reconstruyendo constantemente porque el mundo que intenta interpretar y explicar también se transforma en un movimiento sin cesar.

En la actualidad, más que nunca, se necesitan perspectivas amplias e integradoras y se requieren nuevas teorías y nuevos estudios empíricos para entender esa realidad cambiante a partir de aproximaciones interdisciplinares y multidisciplinares. Es algo que viene exigido porque la ciudad se ha ido convirtiendo en un espacio cada vez más difícil de estudiar y comprender como un sistema integrado, hoy prácticamente inabarcable por una sola disciplina, pero extraordinariamente fértil para la Geografía, que, tomando en consideración todas las transformaciones que ocurren en el tejido urbano y todos los procesos implicados en el cambio (Ravazzoli), puede aportar a las otras disciplinas una base epistemológica y una visión sintética para abordar el análisis de los procesos urbanos.

En este sentido son numerosas y cualificadas las voces que están reclamando la necesidad de repensar lo urbano en un mundo de Urbanización Generalizada o planetaria (Brenner, 2013, Capel, 2010; Espelosín, 2010, García Sánchez, 2013; Hiernaux, 2006) que, incluso, ha suscitado la necesidad de inventar nuevas palabras (Vicente, 2003) para dar nombre a realidades urbanas inéditas y caracterizadas por nuevas lógicas espaciales tendentes hacia la fragmentación y la dispersión ilimitada e informe. Neologismos tales como ciudad-archipiélago, magma city, edge city (Garreau), ciudad hojaldre (García Vazquez), postsuburbia, megápolis, megalópolis, metápolis (Asher, Capel), postmetrópolis y exopolis (Soja, 2001) o pantópolis (García-Bellido), entendida no como la ciudad total sino como la expansión de lo urbano por todo el territorio sin límites definidos, un territorio urbano formado por multitud de micrópolis y metrópolis con innumerables nodos de interconexiones próximas y lejanas (Lindón, 2008).

Tal vez porque «ya no habitamos ciudades sino territorios» (Cacciari, 2010), se está haciendo imprescindible refundar las ciencias urbanas, no sólo la Geografía urbana, pero también ésta, para hallar un nuevo lugar de encuentro desde el cual observar y analizar la realidad de los espacios urbanos. 


\section{BIBLIOGRAFÍA $^{3}$}

BAHAmonde, A. (1993): «La Historia urbana», en Ayer, núm. 10, pp.47-62.

Berdoulay, V. (2001): «Dire la ville comme un tout: la stratégie narrative de Raoul Blanchard à propos de Grenoble», en Berdoulay, V. y Claval, P. (dirs.): Aux débuts de l'urbanisme français. París, L'Harmattan, pp. 83-93.

Berdoulay, V. y Soubeyran, O. (2002): L'Ecologie urbaine et l'urbanisme. Aux fondements des enjeux actuels. París, Éditions de La Découverte. Prefacio de Marcel Roncayolo y traducción de Josefina Gómez Mendoza.

Berdoulay, V. y Soubeyran, O. (2010): «La irrupción de la geografía urbana en la génesis del urbanismo», en Ería, n. ${ }^{\circ}$ 81, pp. 39-57. Gómez MendozA, J. (2009): «Presentación» de Gómez Mendoza, J., pp. 39-42.

Bielza, V. (2011): «El tema de la morfología urbana en la historia del pensamiento geográfico», en Geographicalia, n. ${ }^{\circ}$ 59-60, pp. 27-45.

BORJA, J. y Muxí, Z. (2004): «Una breve y agitada historia entre lo local y lo global», en Urbanismo en el siglo XXI: una visión crítica, Barcelona, Ediciones UPC, pp. 11-14.

Brenner, N. (2013): "Tesis sobre la urbanización planetaria», en Nueva sociedad, n. ${ }^{\circ}$ 243, pp. 38-66.

CACCIARI, M. (2010): La ciudad. Barcelona, Gustavo Gili.

Campesino, A. (1985): «El geógrafo en el planeamiento urbano», en Boletín de la Asociación de Geógrafos Españoles, núm. 2, pp. 24-35.

CAPel, H. (1975): Capitalismo y morfología urbana en España. Barcelona. Los libros de la Frontera.

CAPel, H. (2010): «Urbanización Generalizada, derecho a la ciudad y derecho para la ciudad», en Scripta Nova, vol. XIV, n. ${ }^{\circ} 331$.

Castells, M. (1971): Problemas de investigación en sociología urbana. Madrid, siglo XXI.

Claval, P. (2003): «Métropolisation et globalisation», en Géographie et cultures, n. ${ }^{\circ} 48$.

Delgado, C. (en prensa): «Miradas sobre la ciudad desde la Geografía, la Historia y el Urbanismo. El estado de la cuestión a comienzos del siglo XXI», en Ciudades, n. ${ }^{\circ}$ 19.

Delgado, C., Juaristi, J. y Tomé, S. (2012): Ciudades y paisajes urbanos en el siglo $X X I$. Santander, Ed. Estvdio.

Elden, S., Gregory, D. y Sevilla, Á. (2011): «Espacios del pasado, historias del presente: en torno a los rastros de la historia espacial», en Urban, n. ${ }^{\circ} 1$, pp. 91-114.

GARCíA SÁNCHEZ, R. (2013) Meditación sobre la ciudad actual. Murcia: Edit. UM.

GinTRAC, C. (2013): «Las aportaciones de la geografía radical y la geografía crítica anglosajona a la teoría urbana», en Urban, n. ${ }^{\circ}$ 6, pp. 53-61.

3. Ante la imposibilidad material de reproducir todas las referencias bibliográficas de las y los autores citados, se ha optado por una sucinta selección de menciones consideradas absolutamente imprescindibles. En los demás casos, el nombre del autor o autora, el año de publicación y la temática pueden proporcionar indicios suficientes para encontrar las obras apuntadas. 
Gómez MendozA, J. (1989): «Evolución y análisis de las aportaciones de Geografía Urbana a los estudios Urbanos», en Ciudad y Territorio: Revista de ciencia urbana, 81-82, pp. 87-92.

Gómez MendozA, J. (2004): «Introducción», en MARÍAs, D. (Ed.): Ciudades españolas. Manuel de Terán. Madrid, Real Academia de la Historia, pp. 11-26.

GonzÁlez, J. M. y Rullán, O. (2013): «Informe del Grupo de Geografía Urbana (GGU)», en Lasanta, T. y Martín, J.: La Investigación Geográfica en España (1990-2012). Madrid, Asociación de Geógrafos Españoles, pp. 475-483.

HaRveY, D. (1977): Urbanismo y desigualdad social. Madrid, Siglo XXI.

HieRnAUX, D. (2006): «Repensar la ciudad: La dimensión ontológica de lo urbano», en Liminar. Estudios sociales y humanísticos, vol. IV, n. ${ }^{\circ}$ 2, pp. 7-17.

JUARISTI, J. (2015): «Morfología, funciones y sociedad. La ciudad desde el punto de vista plural de la Geografía Urbana», en Lurralde, n. ${ }^{\circ} 38$, pp. 233-261.

Lindón, A. (2008): «Los giros de la geografía urbana: frente a la pantópolis, la microgeografía urbana», en Scripta Nova, vol. XII, n. ${ }^{\circ} 270$ (62).

Lois, R.C., GonzÁlez, J. M. y Escudero, L. A. (2012): Los espacios urbanos. El estudio geográfico de la ciudad y la urbanización. Madrid, Ed. Biblioteca Nueva.

López Trigal, L. (Ed.) (2003): La ciudad: nuevos procesos nuevas respuestas. León, Asociación de Geógrafos Españoles.

Magnago, V. (2003): «La historia de la planificación urbana como proyecto histórico», en RA. Revista de Arquitectura, n. ${ }^{\circ}$ 5, pp. 45-58.

Mas, R. (1989): «Sobre la Geografía Urbana en España», en Història Urbana I intervenció en el centre històric, Barcelona, Instituto Cartográfico de Cataluña, pp. 163-186.

Morales, G. y MÉndez, B. (2012): «Redes temáticas y políticas urbanas: la red emergente de las 'ciudades tranquilas'», en Ería, n. ${ }^{\circ}$ 88, pp. 153-169.

OCAÑA, C. (1997): «El método histórico en Geografía y el análisis social del espacio urbano», en Baetica. Estudios de Arte, Geografía e Historia, n. ${ }^{\circ}$ 19-1, pp. 251-262.

Precedo, A. (2010): «Un ensayo sobre la evolución de la Geografía Urbana en España: reinventar el estudio de la ciudad», en Geographicalia, n. ${ }^{\circ}$ 58, pp. 5-27.

Quirós, F. (2009): Las ciudades españolas en el siglo XIX, Gijón, Ed. Trea, 430 pp.+ CD. Reedición actualizada de la obra Las ciudades españolas a mediados del siglo XIX, Valladolid, Ámbito, 1991.

RuBio, J. L. (2011): «La nueva Geografía Urbana impulsada por el motor cultural», en $Z$ arquitectura, n. $^{\circ} 16-17$.

SAntos, J. M. (1992): «El desarrollo de la Geografía Urbana en la evolución del pensamiento geográfico contemporáneo», en Espacio, Tiempo y Forma, Serie VI, Geografía, vol. V, pp. 9-40.

Smith, N. (2001): «Nuevo globalismo, nuevo urbanismo», en Documents d'anàlisi geogràfica, n. ${ }^{\circ} 38$, pp. 15-32.

SojA, E. W. (2001): Postmetropolis: Critical Studies of cities and regions. Oxford, Blackwell.

Straszewicz, L. (1981): «Papel del geógrafo en las investigaciones del espacio urbano», en Geographicalia, num. 9, pp.111-116 
TERÁN, M. de (1966): «La ciudad como forma de ocupación del suelo y de organización del espacio» en Revista de Estudios de Administración Local, num. 146, pp. 161-177.

Tomé, S. (2002): «Los estudios de Geografía Urbana histórica en España. Balance y estado de la cuestión», en Historia Contemporánea, n. ${ }^{\circ}$ 24, pp. 83-99.

Valenzuela, M. (Coord.) (2013): Las ciudades españolas en la encrucijada: entre el «boom» inmobiliario y la crisis económica. Madrid, Real Sociedad Geográfica Española.

ViCENTE, J. (2003): «¿Nuevas palabras, nuevas ciudades?, en Revista de Geografía, n. ${ }^{\circ}$ 2, pp.79-103.

Wolch, J. (2003): «Radical openness as method in Urban Geography», en Urban Geography, vol. 24, n. ${ }^{\circ}$ 8, pp. 645-646.

Zoido, F. (2012): «Paisaje urbano. Aportaciones para la definición de un marco teórico, conceptual y metodológico», en Delgado, C., JuARISTI, J. y Tomé, S. (Eds.): Ciudades y Paisajes urbanos en el siglo XXI. Santander. Ed. Estvdio, pp. 13-91.

Zumín, L. (1994): «Los estudios urbanos en la última década: entre la dimensión global y la dimensión local», en Documents d'anàlisi geográfica, n. ${ }^{\circ} 24$, pp. 181-200. 